\section{Estrategias psicosociales utilizadas para el cambio de actitud hacia personas discriminadas por su discapacidad o trastorno mental}

\section{Psychosocial strategies used to change attitudes towards people discriminated against because of their disability or mental disorder}

\section{Resumen}

El presente trabajo permitió realizar una revisión de las estrategias psicosociales relacionadas con el cambio de actitud que suelen ser utilizadas en programas que buscan la modificación actitudinal especialmente en la percepción que tiene la población hacia personas en situación vulnerable de discriminación como suelen ser los discapacitados y los que presentan trastorno mental. Se utilizó la técnica de revisión documental con consultas en bases de datos científicas de Psicología, que arrojó 80 referencias de las cuales 58 hicieron parte del estudio. Los hallazgos encontrados muestran que las estrategias más utilizadas y comunes a los dos tipos de discriminación en programas de reorientación actitudinal son: la educación y el contacto social, que suelen ser combinadas con otros tipos de técnicas.

\section{Palabras clave}

Técnicas psicológicas, cambio de actitud, discriminación, discapacidad y trastorno mental.

\section{Abstract}

This article makes a review of psychosocial strategies related to the change in attitude frequently used in programs aimed at attitudinal changes specifically in the public's perception of persons vulnerable to discrimination many of whom are disabled or have a mental health problem. The method of documentary review was used and scientific psychological databases were searched. The database searches resulted in the retrieval of 80 bibliofraphic references of which 58 were used for the investigation. The findings of the review highlight that the most used strategies in attitudinal reorientation programs, addressing both types of discrimination, are: education and social contact in combination with other techniques.
\end{abstract}

\section{Keywords}

Psychological techniques, change of attitude, discrimination, disability and mental health problems.

\section{Jesús Armando Delgado \\ Meza \\ <jesus.delgado@udi.edu.co> \\ Corporación Universitaria de Investigación y Desarrollo}

Para citar:

Delgado, A. (2015): "Estrategias psicosociales para el cambio de actitud hacia personas discriminadas por su discapacidad o trastorno mental", Revista Española de Discapacidad, $3(2): 27-39$.

Doi: <http://dx.doi.org/IO.5569/23405 I04.03.02.02>

Fecha de recepción: 09-09-20I 5 Fecha de aceptación: O3-I2-20I 5 
Introducción

Diversos programas de intervención psicosocial encaminados a la búsqueda de la reformulación de estereotipos sociales e ideas estigmatizantes han hecho uso de estrategias basadas en distintos paradigmas sobre el cambio de actitudes. El interés por revisar las estrategias de intervención psicológica eficaces para la modificación de actitudes hacia la población de personas discapacitadas (Serrano et al., 20I3) o con trastorno mental (Alvarez y Almeida, 2008) se debe a que se han presentado pocas modificaciones en la percepción que se tiene sobre este tipo de personas, llevando por ende a los sujetos que la poseen a un inadecuado autoconcepto y alteraciones en su socialización, ya que la utilización continua de actitudes negativas se convierten en obstáculos que impiden la integración social de estos dos colectivos. Se trata de una situación en la que fácilmente se ven vulnerados los Derechos Humanos fundamentales, lo que ha llevado a que ésta sea afrontada por diferentes organizaciones internacionales como por ejemplo las Naciones unidas, la Organización Mundial de la Salud, distintas naciones individualmente, grupo de pacientes y de familiares que han visto la necesidad de proponer desde normativas hasta acciones que busquen la oportuna y adecuada integración social de este tipo de personas.

$\mathrm{Y}$ es que la persistencia de prejuicios y actos de discriminación se convierten en una evidencia fehaciente de que la inclusión de personas con discapacidad y/o con trastorno mental a actividades sociales no han mostrado los mejores resultados, tanto en participación, especialmente en lo que tiene que ver con relaciones interpersonales y autoestima por el hecho de enfrentar el riesgo al rechazo, como en aportación social (Palacios, 2008; Hilarion y Koatz, 20I2). Por otra parte, la conservación de actitudes erróneas y conceptualizaciones equívocas están relacionadas con la negación a cambios, resistencias y hasta retrocesos frente a la visión de discapacidad o enfermedad mental (Hernández et al., 2009). Esto se debe particularmente a que ciertos valores y principios de la sociedad se encuentran viciados, lo que requiere que se asuman cambios de actitudes de orden social que permitan abrir espacios, generar más participación y oportunidades de convivencia social.

Precisamente, tratar el tema de los cambios actitudinales refiere retomar el soporte teórico acerca de las actitudes el cual describe que en su estructura según Katz y Stotland (I959), Krech et al. (I962) se destacan tres componentes: el cognitivo, cuyo contenido son creencias y conocimiento del objeto, el afectivo que viene acompañado de la emoción y que suele ser difícil de cambiar y el componente conductual correspondiente a la tendencia o predisposición a actuar. Tanto en la discapacidad como en la enfermedad mental, la estigmatización se ha visto permeada por la influencia de estos tres componentes gracias a la acción de creencias sociales, desafectos y conductas asociadas a la rotulación que la sociedad hace a este grupo de personas, lo cual ha dificultado el desarrollo de los programas de salud mental y la promulgación de sus derechos. Redefinir la idea de inhabilidad y anormalidad en el caso de la discapacidad, y locura y peligrosidad en el caso del trastorno mental serían algunos pasos para combatir este estigma.

La forma como se ha venido actuando para cambiar creencias, afectos y discriminación hacia las personas discapacitadas (Universidad Internacional de Andalucía, 20I4) y/o con trastorno mental (López et al., 2008) ha sido a través de programas basados en la teoría del cambio de actitud que utilizan diversas estrategias ya sea de forma particular o combinada, siendo las más frecuentes el contacto con este tipo de personas, la información sobre temas relacionados con dichas poblaciones y el entrenamiento en habilidades sociales, entre otras.

Es por ello que a través del presente trabajo, se hará una revisión documental que permita escudriñar los procedimientos en cuanto a la utilización de estrategias que inciden sustancialmente en programas sociales e 
intervenciones psicosociales que buscan la modificación en la percepción o el cambio de actitudes en las personas hacia dos sectores poblacionales sensiblemente discriminados: las personas con discapacidad y las personas que presentan algún tipo de trastorno mental.

\section{Metodología}

Este trabajo corresponde a una investigación de tipo cualitativa en la que se utilizó la técnica de revisión bibliográfica o documental con búsqueda en bases de datos científicas de Ciencias Sociales y Humanas tales como Ebsco, Proquest, Psicodoc, Redalyc, Science Direct, PsycInfo, Sociological Abstract y en los índices bibliográficos nacionales y latinoamericanos Publindex y Latindex, la cual arrojó 80 referencias directas e indirectas sobre el tema estrategias para el cambio de actitudes en población adulta que han sido publicadas desde el año I96I hasta el 20I4 tanto en el idioma inglés como en español. Éstas se sometieron a examen de pertinencia quedando 58 , de las cuales, a su vez, las más directamente de orden psicosocial fueron solo 40 . Se definieron a su vez las unidades de estudio, entendidas éstas como los criterios básicos para la creación de fichas de clasificación de la información base de estudio. Estas fueron: título, autores, año de publicación, tema central del documento, ideas relevantes encontradas en el documento y datos a considerar que aportan al objetivo de la investigación; al interior de cada unidad se realizó una categorización con fines de realizar la revisión sistemática.

Finalmente, el estudio documental se desarrolló en cuatro fases: la primera fase, de búsqueda y registro descriptivo de la información de las fuentes; la segunda, interpretativa; la tercera, de sinopsis e integración de los hallazgos y la última fase, de redacción del documento.

Se consideraron como criterios de inclusión de artículos vinculados a la investigación el que se tratase de publicaciones cuyos temas centrales fuesen la exposición de estrategias sobre cambio de actitudes y la utilización de estas en programas orientados a hacer cambiar la visión estereotipada frente a poblaciones discriminadas como son las personas discapacitadas y personas con trastornos mentales, a las cuales se pudiese tener acceso virtual o físico por medio de las bases de datos mencionadas con anterioridad, y cuyas temáticas de análisis correspondan a alguna de las unidades de estudio.

\section{Resultados, análisis y discusión}

Dentro de los estudios encontrados sobre programas propuestos contra la discriminación se evidencia que un número significativo de ellos aplica estrategias de cambio de actitudes que buscan fomentar una percepción saludable y que ayudan a entender la situación de vulnerabilidad en la que se encuentran personas en condición de discapacidad y trastorno mental, permitiendo comprender la importante contribución que pueden llegar a ofrecer a la sociedad así como, también, promover autonomía y calidad de vida estimulando la aceptación y la normalización por parte de las personas objeto de discriminación.

\section{Investigaciones de aplicación de programas de cambio de actitudes hacia la discapacidad}

En el caso de las actitudes colectivas hacia las personas con discapacidad, éstas vienen caracterizadas por un cierto sentido negativo, falsamente mitificadas y cargadas de prejuicios sociales generados en torno a ellas, que conducen a una falsa creencia de que son seres inferiores, incompetentes, ineficaces para enfrentarse a la sociedad, lo que hace que estos estereotipos y prejuicios justifiquen la persistencia de ciertas actitudes hacia las personas con discapacidad (Soto y Vasco, 2008). En ciertos casos, dicha discriminación puede llegar al extremo de que estas personas 
pueden ser excluidas por otras y, de esta forma, negarles sus derechos y una participación activa en la sociedad (Luque y Luque-Rojas, 20II), negándoles el reconocimiento de su condición como parte de las diversidades que compone el mundo y de sus derechos, y el que sean vistas desde su discapacidad y no desde sus múltiples competencias, lo que conlleva a que no se les aproveche para el desarrollo social y económico en una sociedad.

La investigación, propuesta y ejecución de programas de cambio de actitudes hacia la discapacidad viene siendo abordado desde hace más de 20 años y ha sido objeto de estudios específicos (Aguado et al., 2003, 2004; Aguado et al., 2008; Aguado et al., 2006). Sus trabajos han sido basados en la aplicación de técnicas de cambio de actitudes hacia la discapacidad, brindando información directa e indirecta a grupos de personas sobre la discapacidad, estableciendo contacto físico con personas que la presentan y utilizando además ayudas técnicas en sesiones interactivas con discusión guiada, asequible y de fácil realización, que mostraron eficacia y un cierto mantenimiento temporal ya que las actitudes positivas suelen tener un decaimiento estadísticamente significativo. Según estos autores, el contacto estructurado y la información permiten tener un acercamiento a la realidad de la discapacidad, llevando a generar expectativas más ajustadas y a eliminar barreras y prejuicios, afirmación similar a lo encontrado por Gartrell (I986) y Dengra et al. (I99I), quienes adujeron que entre los factores necesarios para producir cambios en las actitudes hacia los sujetos con discapacidad se encuentran la representación de papeles de la condición de discapacidad, el contacto directo con sujetos con discapacidad, el contacto indirecto a través de videos, películas y el trabajo y discusiones en grupo, estas dos últimas de orden informativo.

Más adelante, en un estudio igualmente de Flórez et al. (2009) se realizó una revisión analítica de programas de cambio de actitudes hacia personas con discapacidad, refiriendo que una gran parte de ellos utilizan, bien de forma independiente o combinada distintas estrategias, siendo las más constantes el contacto con personas con discapacidad, la simulación de discapacidades, la información sobre temas relacionados con dicha población, los equipos de trabajo colaborativo, las experiencias en pequeños grupos, los programas de tutoría y el entrenamiento en habilidades interpersonales. Los resultados de la revisión hecha en este estudio muestran que el contacto e información, así como los equipos de trabajo colaborativo, son las técnicas más eficaces para el cambio hacia actitudes más positivas y más aún cuando actúan juntas, tal y como lo enuncian Navas et al. (2004) y Nikolaraizi et al. (2005) al referir que el contacto con personas con discapacidad, sin más, no facilita un cambio de actitudes. La mejora va asociada a un mayor conocimiento de la discapacidad.

El acompañamiento de cualquier técnica tal y como es revelada en los distintos resultados de investigaciones sobre el tema, acentúa la importancia de la educación como el paso inicial en la promoción de las actitudes positivas hacia las personas con discapacidad (Lockhart et al., I998; Shannon et al., 2009).

Por otra parte, teniendo presente que el componente cognitivo no es el único que se considera importante analizar en el cambio de actitudes, el componente afectivo también tiene una incidencia destacable ya que en muchas de las investigaciones la condición de empatía y acercamiento emocional es, sin duda, un aspecto que se observa en programas de intervención, cuyos resultados coinciden con diversos trabajos sobre el tema (Asensio, 2002; Avramidis y Norwich, 2002). Así, el contacto con las personas con discapacidad aumenta las actitudes positivas y se constituye en una variable clave en la configuración de las actitudes hacia ellas (Strohmer et al., I984). Investigaciones como las realizadas por Gómez e Infante (2004) y Newberry y Parish (1987) ratifican que el contacto directo con el discapacitado es un factor que condiciona las actitudes hacia éste, sobre todo cuando existen familiares que la poseen. Las actitudes positivas encontradas en estos estudios estuvieron relacionadas con un contacto cercano y profundo con la persona 
con discapacidad, algo característico y habitual en las relaciones familiares. En este sentido, el grado de vinculación y experiencias con la persona que sufre la discapacidad influye en la percepción, la valoración hacia la discapacidad y el alcanzar concepciones integrales con respecto a los mismos (García y Hernandez, 20I I; Moreno et al., 2006; Novo-Corti et al., 20I I; Wash et al., 2008).

La razón por la cual el contacto y la exposición con personas con discapacidad permiten un cambio en la percepción es porque esto facilita el descubrimiento de los atributos positivos de las personas discapacitadas y desarrollaría una mayor aceptación y entendimiento de las mismas, pudiéndose hacer de forma directa o indirecta y estructurada o no estructurada. Algunos estudios refieren en sus resultados que haciendo uso de contactos no estructurados, la sola interacción produciría el cambio de actitudes (Friedman, I975; Rapier et al., I972); sin embargo, otras investigaciones refieren resultados no significativos o negativos con este tipo de contacto (Stager y Young, I981; Donaldson, I980), las cuales describen que existen factores específicos que contribuyen a cambios actitudinales significativos positivos que no están controlados en las experiencias no estructuradas, existiendo la posibilidad de que se refuercen los estereotipos que las personas "normales" tienen sobre las discapacitadas (Strauch,I970), mientras que en el uso de las interacciones estructuradas con personas discapacitadas, se da lugar a la proporción de imágenes no estereotipadas (Gottlieb, I980) en el que se garantiza la practicidad de las actividades diseñadas, ofreciendo con ello resultados positivos (Bailard et al., I977; Evans, I976; Kierscht, I978; Langer et al., I976; Sedlick y Penta, I975); esto lleva a considerar esta estrategia como una de las más exitosas para producir cambios en la actitudes (Verdugo y Arias, I99I).

García (I997) haciendo uso de una de las técnicas mencionadas por Flórez et al. (2009) puso en práctica un programa de entrenamiento en habilidades interpersonales con un grupo de niños cuya edad estaba dentro del rango de I I-I 5 años. A través de la promoción de habilidades cognitivas de solución de problemas interpersonales se pretendió fomentar la aceptación de la integración de niños ciegos en un ciclo escolar, así como promover la disminución del rechazo a tal integración utilizando como referente un grupo control de niños de condiciones similares al grupo experimental, observándose que tras un programa de entrenamiento, las actitudes hacia la integración se modificaron en la dirección prevista y deseada, evidenciándose la conveniencia de utilizar este tipo de intervenciones con niños pre-adolescentes para incentivar la integración de personas discapacitadas, así como la necesidad de incluir este tipo de actuaciones dentro de los currículos escolares. Años más adelante, Pelechano (2003) utilizó dos programas de habilidades interpersonales orientadas a disminuir las actitudes negativas, concluyendo que las actitudes positivas hacia la integración de las personas con discapacidad son más fáciles de modificar hasta los 30 años y que la autoimplicación desempeña un papel importante en esas actitudes. Esta última afirmación había sido enunciada también por Zabalza (I998) quien señaló que en las nuevas generaciones la minoría de edad y la mayor escolarización son variables para el desarrollo de actitudes positivas hacia las personas que presentan una discapacidad.

Otra evidencia de la variedad de técnicas utilizadas en la búsqueda del cambio de actitud es la simulación como medio de conocimiento bastante directo, profundo y "vivo" sobre la discapacidad, en el sentido de ser experimentada personalmente. La experiencia de aplicación de esta técnica fue efectuada por Verdugo et al. (I994) en un grupo de estudiantes de tercero de secundaria, quienes llegaron a concluir que los tipos de intervención en donde mejor se aplica la afectación sobre los tres componentes de la actitud son: el contacto directo con personas con discapacidad, el conocimiento objetivo de la discapacidad incluyendo por supuesto la simulación o vivencia de la misma. Posteriormente López y López (I997) pretendieron promover una modificación de actitudes que proviniera de la reflexión 
producida por el enfrentamiento entre la actitud previa y la realidad que se le presenta a través de la exposición de las capacidades de las personas con discapacidad y la información sobre la misma, así como también de la simulación de algunas de ellas y el mayor conocimiento sobre las personas que la poseen.

Finalmente, la estrategia contacto social, tal y como se evidenció en descripciones anteriores, así como también en las investigaciones realizadas por Gómez e Infante (2004) y Newberry y Parish (I987), pone de manifiesto que esta forma de interacción efectuada con el discapacitado es un factor que condiciona las actitudes. En el estudio realizado, las actitudes positivas encontradas estarían relacionadas con un contacto cercano y profundo con la persona discapacitada, algo característico y habitual que suele presentarse en las relaciones familiares. En este sentido, el grado de vinculación y experiencias con la persona que sufre la discapacidad influiría en la percepción y valoración personal hacia la discapacidad. Los contextos o ambientes de interacción con personas que presentan discapacidad permiten favorecer el conocimiento sobre estas personas, sus características de discapacidad y la convivencia, evitando con ello exclusiones y desigualdades y haciendo que se produzca un desarrollo justo y solidario que facilite mejores procesos de enseñanza-aprendizaje y valores como el respeto y la tolerancia. Así lo refiere Luque y Luque-Rojas (20II) quien concluye que la discapacidad no es una limitación, ya que el contacto personal refuerza la interacción persona-situación, lo que incrementa o refuerza la sensibilidad social, convirtiéndose en la mejor arma para el desarrollo de actitudes y los valores en una comunidad.

\section{Investigaciones de aplicación de programas de} cambio de actitudes hacia el trastorno mental

De acuerdo a la Organización Mundial de la Salud (2013) debido a la discriminación y estigmatización, las personas con trastornos mentales también sufren violaciones de los derechos humanos, negación de derechos sociales, culturales y económicos, pocas oportunidades de participación en la vida pública y falta de atención a prácticas terapéuticas adecuadas en los centros sanitarios, convirtiéndose en un problema para el desarrollo de programas de salud mental que buscan mejorar los derechos humanos de los enfermos mentales (Guimón, 2004).

La promoción y aplicación de programas sobre el cambio de actitud hacia el trastorno mental como estereotipo social en general, sin recurrir a una clasificación por enfermedad (Eaton et al., 2008), se han extendido debido a la implicación social del tema, a los comportamientos discriminatorios hacia este grupo, a sus repercusiones en la reducción de sus posibilidades en la calidad de vida de quienes se encuentran bajo dicha condición, y en general, a las consecuencias de dichas actitudes en la vida y salud mental comunitaria. Desde las ciencias sociales y humanas, especialmente la Psicologia Social y la Sociología, tres estrategias han sido objeto de estudio y han mostrado tanto resultados positivos como negativos: protesta social, educación y contacto social (López et al., 2008).

Angermeyer y Matschinger (I996) comprobaron cómo la divulgación selectiva en los medios refuerza el estereotipo negativo de la enfermedad mental. Es por ello que la primera estrategia denominada "protesta social" ha tenido unos claros efectos a corto plazo en la lucha contra la estigmatización y la discriminación. Acciones como la solicitud de disminución de imágenes negativas basadas en estereotipos hacia las personas estigmatizadas, la supresión de alguna forma específica de discriminación difundida por los medios de comunicación, han mostrado buenos resultados. Sin embargo, su utilidad a largo plazo ha sido cuestionada, dada la poca utilización de imágenes positivas y la resistencia al cambio de los estereotipos sugeridos a través de los medios, lo que ha hecho que no se mantenga una percepción favorable hacia la población con enfermedad mental (Corrigan y Penn, I999; Corrigan, 200I).

Por otra parte, la estrategia "protesta social" ha ido de la mano con la estrategia "educación” lo que ha suscitado la generación de programas a 
largo plazo que han resultado ser útiles cuando se incluyen no sólo la transmisión pasiva de información sino la exposición de casos reales con presencia de personas que viven la discriminación y una discusión alrededor de la problemática (Rusch et al., 2005; Corrigan y Penn, I999; Estroff et al., 2004; Penn y Couture, 2002), concluyendo que la acción de la educación por sí sola no soluciona el problema de la discriminación, aún más cuando no existen datos explícitos de su capacidad para cambiar creencias o actitudes, sino conductas reales.

Diversos programas se han llevado a cabo a través de la ejecución de proyectos como el denominado Changing minds, Every family in the land emprendida por el Royal College of Psychiatrists del Reino Unido e Irlanda entre el año 1998 y 2003 que puso en marcha una serie de actividades para combatir la estigmatización de personas con enfermedad mental, buscando como objetivo incrementar el conocimiento y la comprensión por parte del público en general y de esta manera disminuir el estigma y la discriminación (Muñoz et al., 2009). Durante cinco años de trabajo en dicho proyecto, se desarrolló un abundante material informativo y audiovisual dirigido a receptores específicos como profesionales en salud, las propias personas con enfermedad mental, padres de familia y profesores, entre otros, destacándose entre las acciones de campaña la creación y divulgación de una guía dirigida a profesionales de la comunicación en donde se recopila información sobre la esquizofrenia, su incidencia, causas, síntomas, diagnósticos y tratamiento.

Por otra parte, la Federación Europea de Asociaciones de Familiares de Enfermos Mentales en el año 2004 inició una campaña con el slogan "Zero Estigma" cuya finalidad fue la disminución del predominio de prejuicios, rechazo y miedo e incentivar la aceptación, información y comprensión. Para ello se valieron de una guía para las campañas locales, exponiendo de manera razonada los métodos que pueden utilizar las asociaciones que forman parte de la Federación para llevar a cabo los proyectos.
Otra iniciativa reconocida es la realizada por la Asociación Mundial de Psiquiatría denominada "Open the doors", desarrollada desde el año I997 en distintos países como España, Canadá, India o China, entre otros, con el objetivo también de aumentar la concientización y los conocimientos acerca de la esquizofrenia y las posibilidades de tratamiento mejorando de esta manera la actitud de las personas hacia aquellas que la han padecido y hacia sus familias. En cada uno de esos países se estableció un grupo en el que participan representantes de organizaciones y personajes reconocidos dedicados a combatir la estigmatización y la discriminación.

Algunos estudios posteriores a la ejecución de los programas anteriormente mencionados han evaluado los efectos de estos programas antiestigma, basados en la representación que de la enfermedad mental hacen los medios de comunicación y las actitudes y percepciones que el público tiene. Los resultados muestran que se han producido algunos cambios positivos moderados, como una reducción de términos y expresiones peyorativas, y un aumento del número de reportajes positivos (Wahl et al., 2002).

Al igual que la estrategia "protesta social", la intervención educativa es otra de las estrategias igualmente efectivas que han ofrecido buenos resultados, sobre todo cuando es continua y de larga duración. Esta ha sido de gran utilidad y ha conllevado a mejores resultados cuando el contenido de los mismos versa más sobre el conocimiento de la enfermedad y de los tratamientos médicos que sobre otro tipo de conocimiento y creencias poblacionales (Crisp, 2000; Kadri y Sartorius, 2005). Además, la iniciativa pedagógica utilizada en la intervención educativa combina el abordaje claramente definido para grupos específicos, como por ejemplo profesionales encargados de procesos educativos, sectores poblacionales concretos como empresarios y personas pertenecientes a barrios que promueven diferentes tipos de mensajes sobre la enfermedad mental y los tipos de creencias a modificar como la idea de peligrosidad y la imprevisibilidad en el caso 
de enfermedades o trastornos graves. Sobre este último aspecto, investigaciones como las realizadas por Casco et al. (I987) con el interés de difundir y estructurar la información existente con respecto al tema de la percepción y las actitudes hacia la enfermedad mental, determinaron que las causales de rechazo hacia el enfermo mental estaban dadas por las características de agresión, irresponsabilidad e impredecibilidad del sujeto y que variables como la educación y jerarquía ocupacional permiten una actitud más favorable hacia el enfermo mental. Estos autores finalmente aseguraron que a medida que los sujetos participantes en el estudio tenían más edad se mostraban menos favorables hacia este tipo de personas. Los resultados de diferentes estudios sobre la educación a grupos sobre discapacidad sugieren que las personas que tienen una menor comprensión de la enfermedad mental son menos propensas a la discriminación y estigma de la enfermedad mental (Brockington et al., I993; Link y Cullen, I986; Link, I987; Roman y Floyd, I98I).

Finalmente, la estrategia de exposición o contacto directo con las personas con enfermedad mental se ha considerado desde hace tiempo como un medio efectivo de lucha contra el estigma, sobre todo cuando este se combina con la educación y se siguen las directrices de lo que se denomina contacto óptimo, es decir, de igual a igual, compartiendo objetivos, no competitivo y siendo supervisado por una autoridad competente (Muñoz et al., 2006). Para potenciar el que se comience a producir este contacto, las propuestas incluyen medidas que favorecen la visualización del problema y la exposición a situaciones de contacto igualitario y positivo a través de modelos con capacidad de influencia, como son los personajes de series televisivas o figuras destacadas de distintos ámbitos que frecuentan los medios de comunicación.

Diversos estudios han definido que el contacto personal suele presentarse como una situación que se puede entender en sentido bidireccional ya que presenta beneficios tanto para las personas que suelen discriminar como a los discriminados, ya que en los primeros, el interactuar con personas con trastorno mental los lleva a conocer y comprender la enfermedad y a los segundos a sentir aceptación y autocontrol. Sin duda alguna este tipo de interacción ofrece los mejores resultados en cuanto a su efecto en la reducción de prejuicios entre grupos sociales (Sherif et al., I961; Johnson et al., I984; Desforges et al., I991), a pesar de lo encontrando inicialmente por Perris y Kemali (I985) quienes estimaron que las actitudes públicas hacia la población con trastornos mentales eran negativas incluso después de una convivencia con ellas posterior a seis meses y las hechas por Angermeyer y Matschinger (I996) quienes destacaron en su estudio que un gran número de personas consideraban como "peligrosos" a los pacientes psiquiátricos cuando son desconocidos, llegando a sugerir como medida fundamental el mantenerlos alejados del trato social. Posteriormente en otras investigaciones como las realizadas por Vezzoli et al. (200I) contrastaron lo anteriormente mencionado con los resultados de su estudio, considerando muy importante tener contactos anteriores con los pacientes psiquiátricos ya que las personas que han conocido e interactuado con un paciente psiquiátrico muestran una actitud más positiva, es decir, menos temor, más integración y oportunidades sociales.

\section{Conclusiones}

El estigma y discriminación que afecta a las personas con discapacidad y enfermedad mental se presenta como un fenómeno en términos de causas y repercusiones, lo que implica que sus posibles soluciones necesiten intervención a nivel social. En esos términos, cuando se pretende cambiar actitudes que buscan específicamente la modificación de creencias estigmatizantes, aunque no es posible determinar el grado de efectividad de algunas estrategias utilizadas, dado que estas mismas en los diferentes programas revisados fueron utilizadas de 
manera simultánea en la mayoría de los casos, se confirma que el aumento de la sensibilización hacia las personas con discapacidad y trastorno mental se debe a la utilización de medidas predominantemente informativas, siendo necesario, como suele suceder en todo proceso educativo, el integrar conocimiento, discusión y, por otra parte, estrategias que faciliten el contacto con las personas que son objeto de discriminación. Estas dos medidas permiten adoptar entornos de aprendizaje que fomentan la participación de individuos tanto discapacitados o con trastorno mental, así como coordinar las posibles variables que intervienen en la mejora actitudinal hacia las personas discriminadas con discapacidad o trastorno mental.
Finalmente, se resalta con respecto a la estrategia de educación o formación, como estrategia común en las dos situaciones de discriminación objeto de estudio, el que esta debe efectuarse de manera adecuada asegurando que un proceso de "psicoeducación social" establezca intervenciones con objetivos claramente segmentados y definidos, brindando información correcta, debidamente articulada durante un tiempo prolongado y que se integren a políticas de salud y estrategias más generales. Tanto las estrategias de formación o educación como contacto social en general, ofrecen buenos resultados y su ejecución no es compleja, permitiendo modificar las concepciones negativas y erróneas acerca de la diversidad y la diferencia. 


\section{Referencias bibliográficas}

Aguado, A. et al. (2003): "Un programa de cambio de actitudes hacia personas con discapacidad en entorno escolar". Análisis y Modificación de Conducta, 29 (I 27): 673-704.

Aguado, A. et al. (2004): "Programas de cambio de actitudes ante la discapacidad". Psicothema, I6 (4): $667-673$.

Aguado, A. et al. (2006): "Programa de cambio de actitudes hacia personas con discapacidad en entornos escolares". Comunicación presentada a las VI jornadas cientificas de Investigación sobre personas con Discapacidad. Salamanca, INICO.

Aguado A. et al. (2008): "Cambio de actitudes hacia la discapacidad con escolares de primaria". Psicothema, 20 (4): 697-704.

Alvarez, L. y Almeida, O. (2008): “Actitudes hacia el trastorno mental y la búsqueda de ayuda psicológica profesional en un grupo de adultos intermedios de la ciudad de Bucaramanga”. Rev.Fac.Med 56 (2).

Alcedo, M. et al. (2013): "Eficacia del contacto e información como técnicas de cambio de actitudes hacia personas con discapacidad en niños de educación primaria". Universitas Psychologica, I2 (2): 493-504.

Angermeyer, M. y Matschinger, H. (I996): “The effect of personal experience with mental illness on the attitude towards individuals suffering from mental disorders". Social Psychiatry and Psychiatric Epidemiology. 3I (6): 32 I-326.

Asensio, J. (2002): "Las actitudes en la reforma: un aspecto de la educación emocional”. Revista Española de Pedagogía, 22I, enero-abril: 5I-64

Avramidis, E. y Norwich, B. (2002): “Teachers attitudes toward integration/inclusion: A review of the literature". European Journal of Special Needs Education, I7 (2): I29-I47.

Bailard, M. et al. (I997): "Improving the social status of mainstreamed retarded children". Journal of educational Psychology, 69: 605-6I I.
Brockington, I. et al. (1993): “The community's tolerance of the mentally ill". British Journal of Psychiatry, 162: 93-99.

Casco, M. et al. (1987): "La actitud hacia la enfermedad mental, una revisión de la bibliografía”. Salud mental, Io (2): 4 I-50.

Corrigan, P. (200I): "Three strategies for changing attributions”, Schizophrenia Bulletin, 27: I87I95.

Corrigan, P. y Gelb, B. (2006): “Three programs that use mass approaches to challenge the stigma of mental illness". Psychiatry, serv, 57 (3): 393-398.

Corrigan, P. y Penn, D. (I999): “Lessons from Social Psychology on Discrediting Psychiatric Stigma”, Am Psychol, 54 (9): 765-776.

Crisp, A. et al. (2000): "Stigmatization of people with mental illness". The British Journal of Psychiatry, I77: 4-7.

Dengra, R. et al. (I99I): "Estudio de las variables que afectan a las actitudes de los maestros hacia la integración escolar de niños con necesidades especiales”. Anuario Español e Iberoamericano de Investigación en Educación Especial. Madrid: CEPE, 47-48.

Desforges, D. et al. (I99I): "Effects of structured cooperative contact on changing negative attitudes toward stigmatized social groups". Journal oI Personality and Social Psychology, 60 (4): 53 I- 544 .

Donaldson, J. (I980): "Cambio de actitudes hacia las personas retrasadas”. Siglo cero, I I 2: 39-38.

Eaton, W. et al. (2008): "The burden of mental disorders”. Epidemiology Rev, 30 (I): I-I4.

Elms, A. (I966): "Influence of fantasy ability on attitude change through role playing". Journal of Personality and Social Psychology, 4 (I): 36-43.

Estroff, S. et al. (2004): "From stigma to discrimination: an analysis of community 
efforts to reduce the negative consequences of having a psychiatric disorder and label”. Schizophrenia Bulletin, 30 (3): 493-509.

Evans, J. (I976): “Changing attitudes toward disabled: an experimental study". Rehabilitation counseling bulletin, I9: 572579 .

Flórez, M. et al. (2009): "Revisión y análisis de los programas de cambio de actitudes hacia personas con discapacidad". Armario de Psicología Clínica y de la Salud, 5: 8 5-98.

Friedman, R. (I975): The peer-peer interaction program: a model program for the integration of severely handicapped youngsters with nondisabled peers, Albertson (Nueva York): Human Resources center.

García, L. (I997): "Fomento de la aceptación de la integración de niños ciegos en ESO”. Comunicación presentada al Simposio Cambios de habilidades interpersonales a lo largo del ciclo vital de las II Jornadas Científicas de Investigación sobre Personas con Discapacidad, Salamanca.

García, L. y Hernandez, O. (20I I): "Actitudes hacia la discapacidad de jóvenes y adultos de Chiapas”. Universitas Psychologica, , Iо (3): $817-827$.

Gartell, A. (I986): “Modifying attitudes toward the handicapped: A review of the literature and methodology". En Jojes, R. L. (Comp): Attitudes an attitude change in special Education. Reston (Virginia): The council for exceptional children.

Gómez, V. e Infante, M. (2004): “Actitudes de los estudiantes de educación hacia la integración de las personas con discapacidad y hacia la educación multicultural". Cultura y Educación I 6 (4): 37 I-383.

Gottlieb, J. (I980): "Improving attitudes toward retarded children by using group discussion". Exceptional children, 47: I06-I I I.

Guimon, J. (2004): La discriminación de los pacientes mentales: un reto para los derechos humanos. Bilbao: Publicaciones de la Universidad de Deusto.
Hernández, R. et al. (2009): "La inclusión de discapacitados intelectuales en el mundo laboral: análisis cualitativo. Estudio de un caso". Revista Latinoamericana de Educación Inclusiva. Escuela de Educación Diferencial Facultad de Ciencias de la Educación 2 (3).

Hilarión, P. y Koatz, D. (20I2): Guía para la integración laboral de personas con trastorno mental. Edición obra social "La Caixa" (en línea). <http://www.incorporasaludmental.org/ images/doc/D_CAS_INI+ENT+EMP_DOCU_ GUIA_0047_Guia_Integ_Laboral.pdf>, acceso IO de diciembre de 2015 .

Johnson, D. et al. (I984): "Goal interdependence and interpersonal attraction in heterogeneous classrooms: A meta analysis”, en Miller, N. y Brewer, M. B. (eds): Groups in contact: The psychology of desegregation. Nueva York: Academic Press.

Kadri, N. y Sartorius, N. (2005): The global fight against the stigma of schizophrenia. PLOS Medicine, 2 (7): 597-599.

Katz, D. y Stotland, E. (I959): “A preliminary statement to a theory of attitude structure and change”. En Koch, S. (Ed): Psychology: a study of science. Formulations of the person and the social context, Nueva York: McGraw-Hill.

Kierscht, M. et al. (I978): Children's attitudes about disabled scale. Psychology department: Moorhead state (manuscrito no publicado).

Krech, D. et al. (1962): Individual in Society. New York: McGraw-Hill.

Langer, E. et al. (I976): “Stigma, staring y discomfort: a novel-stimulus hypothesis". Journal of experimental social psychology, I 2: $45 \mathrm{I}-463$.

Link, B. y Cullen, F. (I986): "Contact with the mentally ill and perceptions of how dangerous the are". Journal of Health and Social Behavior, 27: $230-289$

Link, B. (I987): “Understanding labeling effects in the area of mental disorders: An assessment of the effects of expectations of rejection". American Sociological Review, 52 (I): 96-I I 2. 
Lockhart, R. et al. (I998): "Influence of empathy training to modify attitudes of normal children in physical educativon toward peers with physical disabilities”. Clinical Kinesiology, 52 (2): 35-4I.

López, M. et al. (2008): "La lucha contra el estigma y la discriminación en salud mental. Una estrategia compleja basada en la información disponible". Revista de la Asociación Española de Neuropsiquiatría, IOI (28).

López, M. y López, M. (I997): “Simular la discapacidad. Una técnica para conocer las necesidades educativas especiales y modificar actitudes en la formación del profesorado". Revista electrónica interuniversitaria de formación del profesorado, I (o).

Luque, D. y Luque-Rojas, M. (20 I I): "Conocimiento de la discapacidad y relaciones sociales en el aula inclusiva. Sugerencias para la acción tutorial". Revista Iberoamericana de Educación. 54 (6): I-I2.

Moreno, F. et al. (2006): “Actitudes ante la discapacidad en el alumnado universitario matriculado en materias afines". Revista Iberoamericana de Educación, 40 (5): I 5-25.

Muñoz, M. et al. (2006): Informe de propuestas específicas para la reducción del estigma social en las personas con enfermedad mental grave y crónica, Madrid: Facultad de psicología. Universidad Complutense de Madrid.

Muñoz, M. et al. (2009): Estigma y enfermedad mental. Análisis del rechazo social que sufren las personas con enfermedad mental, Madrid: Editorial Complutense.

Muñoz, J. et al. (20I3): “Análisis de las actitudes, de los jóvenes trabajadores del sector textil hacia la discapacidad: diferencias por razón de género". Revista de Investigación Educativa, 3I (I): 93-II 5 .

Navas, L. et al. (2004): "Algunas variables predictoras de las actitudes del alumnado ante la integración escolar”. Revista de Psicología Social, I9 (2): I 59-I7I.

Newberry, M.K. y Parish, T. (I987): “Enhancement of attitudes toward handicapped children through social interactions". Journal of Social Psychology, I 27 (I): 59-62.

Nikolaraizi, M. et al. (2005): “A cross - cultural examination of typical developing children;s attitudes toward individuals with special needs". International Journal of Disability. Development and Education, 52 (2): IOI-I I9.

Novo-Corti, I. et al. (20I I): “Análisis de las actitudes de los jóvenes universitarios hacia la discapacidad: un enfoque desde la teoría de la acción razonada". RELIEVE, 2 (I7).

Organización Mundial de la Salud, OMS (2013): Informe sobre la salud en el mundo 2013: investigaciones para una cobertura sanitaria universal. Luxemburgo (en línea). <http://apps.who.int/iris/ bitstream/I0665/8 5763/I/978924069I223_ spa.pdf $>$, acceso I I de diciembre de 2015 .

Palacios, A. (2008): El modelo social de discapacidad: orígenes, caracterización y plasmación en la convención internacional sobre los derechos de las personas con discapacidad, Madrid: Ediciones Cermi (en línea). <http://www.cermi.es/es-ES/ ColeccionesCermi/Cermi.es/Lists/Coleccion/ Attachments/64/Elmodelosocialdediscapacidad. pdf $>$, acceso I I de diciembre de 20 I 5 .

Pelechano, V. (2003): "El estudio de la discapacidad desde el punto de vista psicosocial”. Análisis y Modificación de conducta, 29 (I25): 327-393.

Penn, D. et al. (I994): "Dispelling the stigma of schizophrenia: what sort of information is best?”. Schizophrenia Bulletin, 20: 567-74.

Penn, D. y Couture, S. (2002): “Strategies for reducing stigma towards persons with mental illness". World Psychiatry, I (I): 20-2I.

Perris, C. y Kemali, D. (1985): "Focus on the Italian psychiatric reform: an introduction". Acta Psychiatr Scand Suppl, 3 16: 9-I4.

Rapier, J. et al. (1972): “Changes in children's attitudes toward the physically handicapped". Exceptional children, 39: 219-223.

Roman, P. y Floyd, H. (I98I): "Social acceptance of psychiatric illness and psychiatric treatment”. Social Psychiatry, I6: I6-2I. 
Rusch, N. et al. (2005): "Mental illness stigma: concepts, consequences and initiatives to reduce stigma”. European Psychiatry, 20: 529-539.

Sedlick, M. y Penta, J. (I975): “Changing nurse attitudes toward quadriplegics through use of television", Rehabilitation literature, 36: 274-278.

Serrano, C. P. et al. (20I3): "Barreras contextuales para la participación de las personas con discapacidad física: Discapacidad y barreras contextuales". Revista de la Universidad Industrial de Santander. Salud, I (45): 4I5I (en línea). <http://www.scielo.org.co/ scielo.php?script=sci_arttext\&pid=So I 2 I 080720I3000I00006\&lng=en\&tlng=es >, acceso I I de diciembre de 2015.

Shannon, C. et al. (2009): "The effect of contact, context, and social power on undergraduate attitudes toward persons with disabilities". Journal of rehabilitation, 75 (4): I I-I 8.

Sherif, M. et al. (196I): The Robbers Cave Experiment: Intergroup Conflict and Cooperation. Norman, Oklahoma: Book Exchange, University of Oklahoma.

Soto, V. y Vasco, E. (2008): "Representaciones sociales y discapacidad". Hologramática, 8 , (VI): 3-22.

Stager, S. y Young, R. (I98I): "Intergroup contact and social outcomes for mainstreamed EMR adolescents", American Journal of mental deficiency, 85:497-503.

Strauch, J. (1970): "Social contact as a variable in the expressed attitudes of normal adolescents toward EMR pupils", Exceptional Children, 36: 495-500.

Strohmer, D. et al. (I984): “Attitudes toward persons with disability: An examination of demographic factors, social context and specific disability". Rehabilitation Psychology, 3 (29):

I 3 I-I 45 .

Universidad Internacional de Andalucía (20I4):

$I^{a}$ Jornada sobre maltrato a las personas con discapacidad, Sevilla: Universidad Internacional de Andalucía (en línea). <http://dspace.unia.es/ bitstream/handle/I0334/337I/Irajornadamalt rato.pdf? sequence $=\mathrm{I}>$, acceso $\mathrm{I} 7$ de diciembre de 2015 .

Verdugo, M. y Arias, B. (I99I): "Evaluación y modificación de las actitudes hacia los minusválidos”. Revista de Psicologia General y Aplicada, 44 (I): 95-IO2.

Verdugo, M. et al. (1994): Actitudes hacia las personas con minusvalía. Madrid: Ministerio de Asuntos sociales.

Vezzoli R. et al. (200I): “Attitude toward psychiatric patients: a pilot study in a northern town”. European Psychiatry, I 6: 45 I-458.

Wahl, O. et al. (2002): "Newspaper coverage of mental illness: is it changing?" Psychiatric Rehabilitation Skills, 6 (I): 9-3I.

Wash, M. et al. (2008): "Attitudes of University Students toward individuals with exceptionalities and inclusive Practices: A Baseline Analysis of students Enrolled in the Introductory Course". NERA conference proceedings, (22).

World Health Organization, WHO (2OII): World report disability (en línea). <http://whqlibdoc. who.int/publications/20I I/97892406852I 5 eng.pdf>, acceso 30 de noviembre de 2015.

Zabalza, M. (I998): "Evaluación de actitudes y valores”. En Medina, A. et al. (Eds.): Evaluación de los procesos y resultados de aprendizaje de los estudiantes. Madrid: Editorial UNED. 